\title{
Plasma Concentration of the Lipid Peroxidation (LP) Biomarker 4-Hydroxynonenal (4-HNE) in Benign and Cancer Patients
}

\author{
MAARET ESKELINEN ${ }^{1 *}$, IINA SAIMANEN ${ }^{1 *}$, RIIKA KOSKELA ${ }^{2}$, \\ ANU HOLOPAINEN ${ }^{3}$, TUOMAS SELANDER ${ }^{4}$ and MATTI ESKELINEN ${ }^{1 *}$ \\ ${ }^{1}$ Department of Surgery, Kuopio University Hospital and School of Medicine, \\ University of Eastern Finland, Kuopio, Finland; \\ ${ }^{2}$ Department of Anaesthesiology and Intensive Care, Kuopio University Hospital, Kuopio, Finland; \\ ${ }^{3}$ Department of Clinical Chemistry, Eastern Finland Laboratory Center, \\ Kuopio University Hospital, Kuopio, Finland; \\ ${ }^{4}$ Science Service Center, Kuopio University Hospital, Kuopio, Finland
}

\begin{abstract}
Background/Aim: The present study investigated the plasma concentration of the lipid peroxidation (LP) biomarker 4-hydroxynonenal (4-HNE) in benign and cancer patients having the rectus sheath block (RSB) analgesia after midline laparotomy. Plasma concentrations of catalase (CAT) and malondialdehyde (MDA) were used as a reference. Patients and Methods: This study assessed three LP biomarkers; CAT, MDA and 4-HNE and compared the plasma levels to the patient satisfaction 24 h postoperatively ( $\mathrm{SFS}_{24}$; $0=$ fully unsatisfied; $10=$ fully satisfied); the overall pain at rest $\left(N R S_{r}\right)$ and when pressing the wound at 20 Newton force $\left(N R S_{p}\right)$ were surveyed and filed on a 11-point numeric rating scale at 24 following surgery (NRS; $0=$ no pain; $10=$ worst pain). There were 56 patients in the study, of whom 12 were excluded due to missing plasma samples. The final study cohort consisted of 15 patients with benign disease and 29 patients with cancer. Results: The RSB analgesia enhanced significantly the $S_{2} S_{24}$ scores in the study groups $(p=0.001)$. The plasma 4$H N E$ decreased immediately after operation (POP1) and the postoperative decrease between the preoperative and the POPI values in the 4-HNE marker were statistically significant
\end{abstract}

This article is freely accessible online.

*These Authors contributed equally to this study.

Correspondence to: Matti Eskelinen, MD, Ph.D., School of Medicine, University of Eastern Finland, P.O. Box 100, FI-70029 KYS, Finland. Tel: +358 17173311, Fax: +358 17172611, GSM: +358400969444, e-mail: matti.eskelinen@kuh.fi

Key Words: Benign disease, cancer, lipid peroxidation, plasma 4hydroxynonenal, pain score following surgery. $(p<0.001)$. The individual plasma 4-HNE and MDA concentration correlated significantly in benign and cancer patients ( $r=0.413, p<0.001)$. Conclusion: The present study confirms the applicability of the plasma biomarker 4-HNE to cast further light on the postoperative pain in midline laparotomy patients.

Failure in antioxidant defenses could lead to formation of reactive oxidative species (ROS) and lipid peroxidation (LP) (1-9). The uncontrolled ROS is called oxidative stress (OS) and is a common feature in cancer cells (1). OS in cancer cells could be demonstrated by an increase in OS markers and ROS in cancer cells can be counteracted by substances known as antioxidants: vitamin $\mathrm{E}$ and $\mathrm{C}$, flavonoids and carotenoids $(10,11)$. The most important antioxidative enzymes are catalase (CAT) $(12,13)$, glutathione peroxidase (GPX1) $(4,14)$ and superoxide dismutase (SOD1) $(15,16)$. The role of LP in etiology of cancer, neurodegenerative and cardiovascular diseases, has motivated researchers to clarify the mechanism of LP and to develop biomarkers $(7,17-19)$. LP proceeds by three mechanisms; (i) free radical oxidation, (ii) free radical independent non-enzymatic oxidation, and (iii) enzymatic oxidation $(1,2)$. Polyunsaturated fatty acids (PUFAs) and cholesterol are oxidized through enzymatic and non-enzymatic routes. Malondialdehyde (MDA) and 4Hydroxynonenal (4-HNE) are the ROS that cause OS in cells, targeting the subcellular structures to form covalent protein adducts $(1,2)$. 4-HNE is an $\alpha, \beta$-unsaturated hydroxyalkenal that is produced by LP in cells. 4-HNE has 3 reactive groups: an aldehyde, a double-bond at carbon 2, and a hydroxy group at carbon $4(1,2)$.

As far as we know, the differences in the LP stress biomarker 4-HNE plasma concentrations in midline 


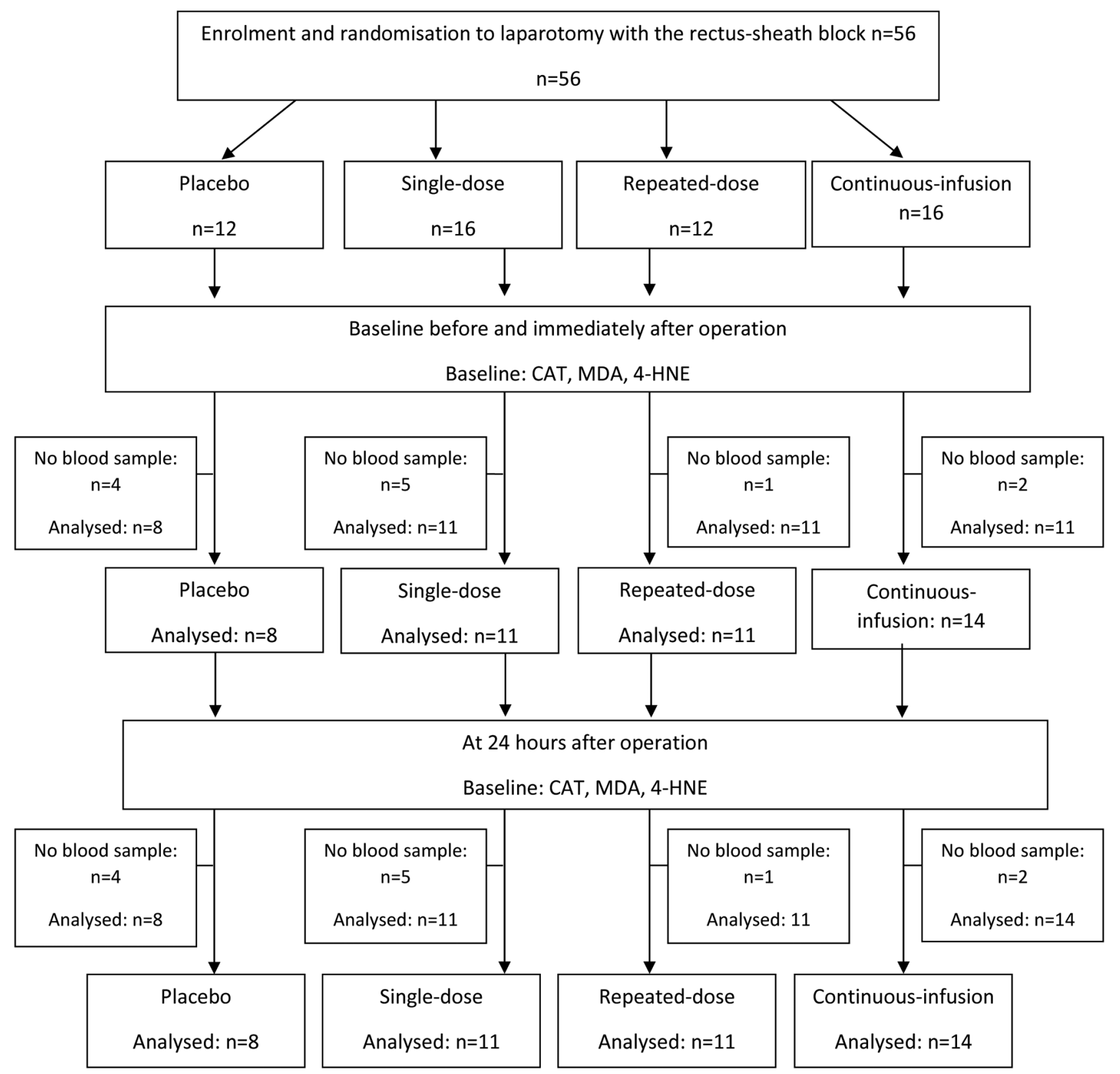

Figure 1. Study flowchart.

laparotomy in patients with benign disease versus cancer is unknown. Therefore, the present study investigated the 4-HNE plasma concentrations in patients with benign disease versus cancer following midline laparotomy. Our aim was to determine plasma 4-HNE levels in patients with benign disease versus patients with cancer and to determine the association between plasma 4-HNE concentrations and the patient satisfaction $24 \mathrm{~h}$ following surgery $\left(\mathrm{SFS}_{24}\right)$, as well as the overall pain at rest $\left(\mathrm{NRS}_{\mathrm{r}}\right)$ and at $2 \mathrm{~kg}$ pressing $\left(\mathrm{NRS}_{\mathrm{p}}\right)$.

\section{Patients and Methods}

Patients in the study. The study was approved by the Ethics Committee of the Northern Savo Hospital District, Kuopio, Finland (DNRO 120/2011, November 11, 2011) and was registered in the EudraCT database (EudraCT number 2011-005136-25, Consort diagram, Figure 1) and in the ClinicalTrials.gov database (ClinicalTrials.gov Identifier: NCT02869841). The study protocol and inclusion/exclusion criteria of the study patients were detailed in the previous report by Purdy et al. (3). 
Eskelinen et al: Lipid Peroxidation in Benign and Cancer Patients

Table I. The clinical data of the four study groups. Data are mean ( \pm standard deviation) or number of cases.

\begin{tabular}{|c|c|c|c|c|c|}
\hline Variable & $\begin{array}{c}\text { Control } \\
\mathrm{n}=8\end{array}$ & $\begin{array}{c}\text { Single } \\
\mathrm{n}=11\end{array}$ & $\begin{array}{c}\text { Repeated } \\
\mathrm{n}=11\end{array}$ & $\begin{array}{c}\text { Continuous } \\
\mathrm{n}=14\end{array}$ & $p$-Value \\
\hline Age, years & $62.6(14.3)$ & $60.8(12.6)$ & $63.3(10.8)$ & $58.0(10.1)$ & 0.74 \\
\hline Gender female/male & $4 / 4$ & $7 / 4$ & $9 / 2$ & $12 / 2$ & 0.22 \\
\hline Height, cm & $166.6(8.6)$ & $168.4(7.9)$ & $165.7(7.2)$ & $164.3(6.6)$ & 0.62 \\
\hline Weight, kg & $78.6(11.8)$ & $83.7(12.8)$ & $67.8(13.7)$ & $68.8(10.6)$ & 0.007 \\
\hline BMI, $\mathrm{kg} / \mathrm{m}^{2}$ & $28.3(3.8)$ & $29.6(4.4)$ & $24.6(4.3)$ & $25.7(4.9)$ & 0.03 \\
\hline Time in the operative room, $\min$ & $229.4(113.4)$ & $274.9(148.4)$ & $235.7(112.0)$ & $279.7(178.5)$ & 0.85 \\
\hline Operative time, $\min$ & $209.6(141.2)$ & $221.8(156.4)$ & $154.4(95.0)$ & $253.3(168.9)$ & 0.55 \\
\hline Perioperative-bleed, $\mathrm{ml}$ & $696(741)$ & 822(906) & 697 (967) & $1340(928)$ & 0.31 \\
\hline ASA $1 / 2 / 3 / 4$ & $0 / 6 / 2 / 0$ & $0 / 7 / 3 / 1$ & $0 / 5 / 6 / 0$ & $2 / 7 / 5 / 0$ & 0.43 \\
\hline $\mathrm{NRSr}$ & $2(1-6)$ & $2(0-4)$ & $2(0-3)$ & $1(0-4)$ & 0.41 \\
\hline NRSp & $5(3-9)$ & $5(1-9)$ & $3.5(1-7)$ & $4(0-7)$ & 0.42 \\
\hline SFS24 & $8(7-8)$ & $9(8-10)$ & $9.5(8-10)$ & $10(8.3-10)$ & 0.001 \\
\hline Length of the skin incision(s), mm & $27.2(6.6)$ & $24.4(7.8)$ & $24.2(7.9)$ & $29.7(7.3)$ & 0.31 \\
\hline Type of disease & & & & & 0.32 \\
\hline Benign $(n=15)$ & 1 & 3 & 5 & 6 & \\
\hline GI cancer $(n=9)$ & 3 & 2 & 2 & 2 & \\
\hline Gyn cancer $(n=17)$ & 3 & 4 & 4 & 6 & \\
\hline Other cancer $(n=3)$ & 1 & 2 & 0 & 0 & \\
\hline
\end{tabular}

GI: Gastrointestinal tract; Gyn: gynaecological; BMI: body mass index; ASA: American Society of Anesthesiologists physical status score; NRS: 11-point numeric rating scale $24 \mathrm{~h}$ after surgery; NRSr: NRS at rest; NRSp: NRS under $2 \mathrm{~kg}$ pressure to the wound area; SFS24: satisfaction following surgery; RSB: rectal sheath block.

Plasma biomarkers and outcome measures. The primary outcome measures were the plasma concentrations of CAT, MDA and 4-HNE biomarkers measured at three time points with high-sensitivity assays: before (PRE), immediately after (POP1) and $24 \mathrm{~h}$ postoperatively (POP2) in the control group versus three active groups. The patient satisfaction at $24 \mathrm{~h}$ postoperatively were filed on a 11-point numeric rating scale $\left(\mathrm{SFS}_{24} ; 0=\right.$ fully unsatisfied; $10=$ fully satisfied) and the overall pain at rest $\left(\mathrm{NRS}_{\mathrm{r}}\right)$ and at $2 \mathrm{~kg}$ pressing $\left(\mathrm{NRS}_{\mathrm{p}}\right)$ was surveyed and filed on a 11-point numeric rating scale $24 \mathrm{~h}$ following surgery (NRS; $0=$ no pain; 10=worst pain).

Samples collection. EDTA-blood samples were taken at the prespecified time-points and centrifuged at $1,000 \times g$ for $15 \mathrm{~min}$. Plasma was separated and stored frozen at $-70^{\circ} \mathrm{C}$ until further analysis. The plasma CAT assays were performed using enzymelinked immunosorbent assay ELISA Kit (Cloud-Clone Corporation, Katy, TX, USA). Plasma MDA concentrations were determined by the MDA ELISA Kit (E-EL-0060, Elabscience, Houston, TX, USA). The manufacturer's intra-assay and inter-assay Cvs were 5.6\% and $6.28 \%$, respectively. The plasma 4-hydroxynonenal (4-HNE) concentrations were measured at three time points with 4Hydroxynonenal Elisa kit (Cat\# 9705, Alpha Diagnostic Intl., San Antonio, TX, USA): before (PRE), immediately after (POP1) and $24 \mathrm{~h}$ postoperatively ( $\mathrm{POP} 2)$. The manufacturer's intra- and interassay Cvs were $<8 \%$ and $<10 \%$.

Statistical analysis. Data are presented as means and standard deviations or frequencies and percentages, where appropriate. Differences in baseline characteristics between groups were tested by Fisher's exact test and in the case of continuous data, the analysis was performed by Kruskal-Wallis test. Group differences at three time points were tested by the Wilcoxon signed rank test and the Kruskal-
Wallis test. The plasma CAT, MDA and 4-HNE concentrations and differences in the patients with benign diseases and cancer were tested by the Mann-Whitney $U$-test. Linear mixed effect (LME) model was used to test time effect. In LME analysis plasma concentrations were $\log$ transformed. The results of the laboratory measurements are presented as median concentrations with interquartile range as distributions were right-skewed. $p$-Values under 0.05 were considered statistically significant. The Spearman's method was used to test for correlation for plasma 4-HNE concentrations versus MDA concentrations. Data were analyzed by IBM SPSS statistical software (SPSS Statistics 26.0.0.1; IBM, Armonk, NY, USA).

\section{Results}

Patient data. There were 56 patients, of whom 12 were excluded due to missing plasma samples. The final study cohort consisted of 15 patients with benign disease and 29 patients with cancer (Table I). There were significantly less benign patients than cancer patients in the control group (one benign patient versus seven cancer patients, benign-cancer ratio $=14.3 \%$ ) than that in the RSB study groups combined (14 benign patients versus 22 cancer patients, benign-cancer ratio $=63.6 \%$ ). The RSB analgesia enhanced significantly the median $\mathrm{SFS}_{24}$ scores in the following order: control, single dose, repeated dose and continuous dose group $(8,9,9.5$ and 10 , respectively; $p=0.001$, Table I). There were no statistically significant differences detected in the $\mathrm{NRS}_{\mathrm{r}}$ and $\mathrm{NRS}_{\mathrm{p}}$ scores between the four study groups $\left(\mathrm{NRS}_{\mathrm{r}} p=0.41\right.$ and $\mathrm{NRS}_{\mathrm{p}} p=0.42$, Table I). 
Table II. The plasma catalase (CAT), malonidialdehyde (MDA) and 4-hydroxynonenal (4-HNE) concentrations in the four study groups. Plasma concentrations were measured before (PRE), immediately after (POP1) and 24 h after (POP2) surgery. Median (interquartile range) concentrations are shown. The Kruskall-Wallis test was used.

\begin{tabular}{|c|c|c|c|c|c|}
\hline Marker & Control & Single & Repeated & Continuous & $p$-Value \\
\hline CAT $(\mathrm{pg} / \mathrm{ml})$ & & & & & 0.506 \\
\hline PRE & $0.95(0.67-2.36)$ & $0.74(0.45-1.58)$ & $0.61(0.41-1.09)$ & $0.61(0.43-1.17)$ & 0.407 \\
\hline POP1 & $1.36(0.89-3.71)$ & $1.89(0.78-2.93)$ & $1.53(0.78-2.17)$ & $1.39(0.84-2.03)$ & 0.907 \\
\hline POP2 & $1.17(0.58-1.65)$ & $0.86(0.60-1.73)$ & $1.15(0.77-1.50)$ & $1.06(0.83-1.92)$ & 0.891 \\
\hline MDA (ng/ml) & & & & & 0.448 \\
\hline PRE & $642(480-1241)$ & $872(670-1132)$ & 795 (685-907) & $983(688-1329)$ & 0.478 \\
\hline POP1 & $541(321-1165)$ & $753(576-801)$ & $555(430-698)$ & $638(525-909)$ & 0.484 \\
\hline POP2 & $480(413-946)$ & $651(533-836)$ & $577(343-799)$ & $601(373-784)$ & 0.767 \\
\hline 4-HNE (pg/ml) & & & & & 0.608 \\
\hline PRE & $245(211-562)$ & $270(153-327)$ & $264(209-356)$ & $274(177-363)$ & 0.979 \\
\hline POP1 & $207(164-289)$ & $242(122-302)$ & $203(167-409)$ & $177(106-322)$ & 0.856 \\
\hline POP2 & $153(56-187)$ & $132(75-155)$ & $119(52-191)$ & $107(90-206)$ & 0.862 \\
\hline
\end{tabular}

Linear mixed model $p$-values for interaction time group are in bold.

Plasma CAT, MDA and 4-HNE concentrations in the RSB study groups. There were no differences detected in the CAT, MDA and 4-HNE concentrations between the four study groups preoperatively and following surgery (Table II). Although, the patients in the control group had a trend for a slightly higher median CAT levels preoperatively compared to the single dose group, repeated dose and continuous dose groups $(0.95 \mathrm{pg} / \mathrm{ml}$ versus $0.74,0.61$ and $0.61 \mathrm{pg} / \mathrm{ml}$, respectively, Table II). The patients in the control group had a trend for lower median MDA concentrations preoperatively and following surgery (POP1), compared to RSB study groups (control group, 642/541 ng/ml versus single dose; $872 / 753 \mathrm{ng} / \mathrm{ml}$ and repeated dose; 795/555 $\mathrm{ng} / \mathrm{ml}$ and continuous infusion; 983/638 ng/ml, Table II). There were no significant differences detected in the preoperative 4-HNE concentrations in RSB study groups and between control groups (Table II). However, the patients in the control group and in the RSB study groups had a trend for lower 4-HNE concentrations following surgery and the most significant postoperative decrease was in patients in the repeated dose and continuous dose groups (Table II).

The alteration of plasma CAT, MDA and 4-HNE levels following surgery. The alteration of plasma CAT, MDA and 4HNE marker levels in the study groups combined are shown in Table III. There were statistically significant increases in the median plasma concentrations of CAT preoperatively (PRE) and immediately after operation (POP1) $(52.5 \%$ increase, $p<0.001$, Table III). Then the median plasma concentrations of CAT marker decreased $24 \mathrm{~h}$ postoperatively (POP2) ( $p=0.001$, Table III). The median plasma concentrations of MDA decreased $24 \mathrm{~h}$ postoperatively (POP2) and the decrease was significant (31.6\% decrease, $p<0.001$, Table III). There was significant decrease in the
Table III. The alteration of the plasma catalase (CAT), malonidialdehyde (MDA) and 4-hydroxynonenal (4-HNE) concentrations following surgery in all groups combined (all patients). Plasma concentrations were measured before (PRE), immediately after (POP1) and 24 hostoperatively (POP2). Median (interquartile range) concentrations are shown

\begin{tabular}{lccr}
\hline Marker & All patients & Alteration & $p$-Value* \\
\hline CAT (pg/ml) & & & \\
$\quad$ PRE & $0.66(0.48-1.17)$ & & \\
POP1 & $1.39(0.84-2.43)$ & PRE vs. POP1 & $<0.001$ \\
POP2 & $1.03(0.70-1.57)$ & POP1 vs. POP2 & 0.001 \\
MDA (ng/ml) & & & \\
PRE & $870(625-1118)$ & & \\
POP1 & $620(432-860)$ & PRE vs. POP1 & $<0.001$ \\
POP2 & $595(410-831)$ & POP1 vs. POP2 & 0.047 \\
4-HNE (pg/ml) & & & \\
PRE & $265(201-348)$ & & \\
POP1 & $203(133-317)$ & PRE vs. POP1 & 0.001 \\
POP2 & $132(80-175)$ & POP1 vs. POP2 & $<0.001$ \\
\hline
\end{tabular}

*Wilcoxon signed-rank test.

median plasma concentrations of MDA preoperatively and immediately after operation ( $p<0.001$, Table III). Also, the median plasma concentrations of 4-HNE decreased $24 \mathrm{~h}$ following surgery (POP2) and the decrease was statistically significant (50.2\% decrease, $p<0.001$, Table III).

Plasma CAT, MDA and 4-HNE concentrations in benign versus cancer patients. The median (interquartile range) plasma concentrations of CAT, MDA and 4-HNE did not differ significantly between the benign and cancer patients preoperatively (Table IV). Patients in the cancer group had a trend for higher median CAT levels postoperatively (POP1) 
Table IV. The plasma catalase (CAT), malonidialdehyde (MDA) and 4hydroxynonenal (4-HNE) concentrations in the patients with benign diseases $(n=15)$ and in those with cancer $(n=29)$. Plasma concentrations were measured before (PRE), immediately after (POP1) and $24 h$ postoperatively (POP2). Median (interquartile range) concentrations are shown. Mann-Whitney U-test was used.

\begin{tabular}{lccc}
\hline Marker & Benign & Cancer & $p$-Value \\
\hline CAT (pg/ml) & & & $\mathbf{0 . 7 1 8}$ \\
PRE & $0.62(0.41-1.17)$ & $0.73(0.48-1.30)$ & 0.407 \\
POP1 & $1.09(0.78-2.50)$ & $1.46(1.13-2.36)$ & 0.304 \\
POP2 & $0.83(0.75-1.65)$ & $1.12(0.68-1.50)$ & 0.593 \\
MDA (ng/ml) & & & $\mathbf{0 . 3 5 3}$ \\
PRE & $920(716-1326)$ & $868(571-1037)$ & 0.211 \\
POP1 & $852(614-955)$ & $589(392-730)$ & 0.021 \\
POP2 & $697(407-868)$ & $560(411-758)$ & 0.383 \\
4-HNE (pg/ml) & & & $\mathbf{0 . 0 3 7}$ \\
PRE & $266(224-281)$ & $263(191-358)$ & 0.970 \\
POP1 & $218(184-334)$ & $202(115-310)$ & 0.328 \\
POP2 & $107(66-187)$ & $133(86-174)$ & 0.495 \\
\hline
\end{tabular}

Linear mixed model $p$-values for interaction time group are in bold.

compared to benign patients (Table IV). There was a statistically significant difference in the median MDA concentrations between benign and cancer patients immediately following surgery (POP1), with the cancer patients showing significantly lower MDA concentrations postoperatively ( $p=0.021$, Table IV). There was a statistically significant decrease in 4-HNE concentrations postoperatively in benign and cancer patients (Figure 2 and Table IV). In addition, the time effect in the linear mixed model in plasma 4-HNE was statistically significant (Table IV).

Correlation of $\mathrm{SFS}_{24}, N R S_{r}$ and $N R S_{p}$ versus the $L P$ biomarkers $M D A$ and 4-HNE. $\mathrm{NRS}_{\mathrm{r}}$ correlated significantly to 4 -HNE plasma concentrations $(p=0.026)$. However, there was no significant correlation between $\mathrm{SFS}_{24}$ or $\mathrm{NRS}_{\mathrm{p}}$ versus MDA or 4-HNE plasma concentrations. The median (interquartile range) plasma concentrations of MDA correlates significantly to $4-\mathrm{HNE}$ concentrations in benign and cancer patients (Figure 3, $\mathrm{r}=0.347, p<0.001$ ).

\section{Discussion}

Albeit the surgical procedure is a common cause of LP in patients with midline laparotomy, the assessment of LP stress biomarker 4-HNE concentrations is rarely reported (1). Especially, the 4-HNE plasma levels versus the patient satisfaction and the NRS pain scores following surgery in midline laparotomy patients is unknown. To cast further light on this issue, the present study was designed to conduct a detailed analysis of the i) Plasma CAT, MDA and 4-HNE concentrations in benign versus cancer patients, ii) The

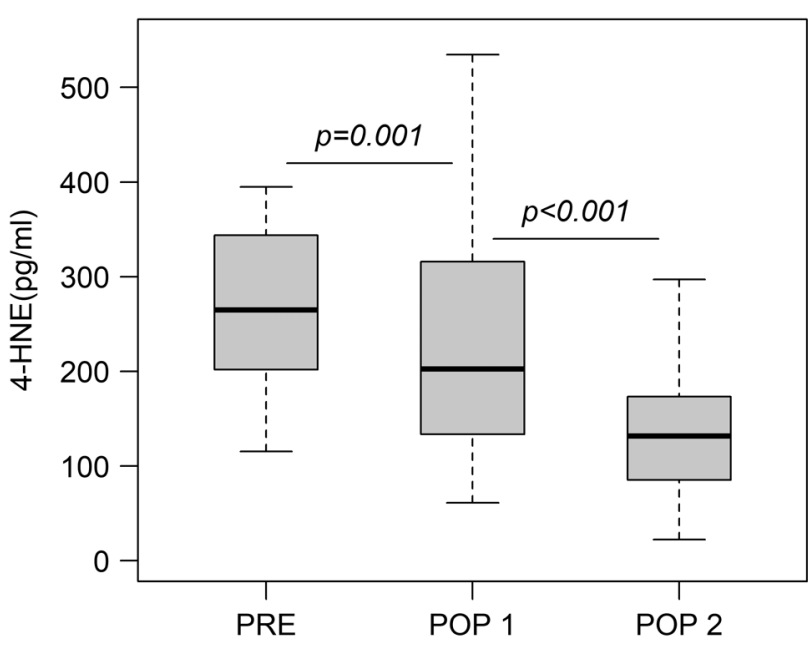

Figure 2. Plasma median 4-hydroxynonenal (4-HNE) concentrations in benign and cancer patients preoperatively, immediately after operation and 24 h after operation. $p \leq 0.001$ using the Wilcoxon signed-rank test.

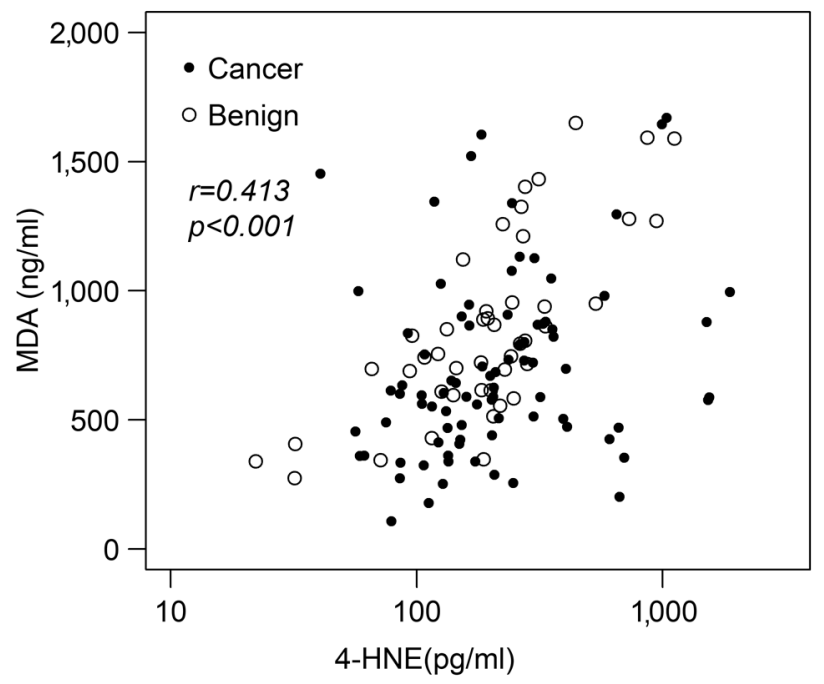

Figure 3. Plasma malonidialdehyde (MDA) concentrations versus plasma 4-hydroxynonenal (4-HNE) concentrations in benign and cancer patients shown as Jitterplot. Spearman's $r=0.413, p<0.001)$.

alteration of plasma CAT, MDA and 4-HNE levels in RSB study groups, and iii) Plasma CAT, MDA and 4-HNE concentrations in RSB study groups, to establish whether these plasma biomarkers could improve the detection of OS or LP stress following surgery. The original study hypothesis was that RSB analgesia could lower the pain and enhance satisfaction in midline laparotomy patients. Therefore, the main aim of the study was to assess the relation between plasma 4-HNE concentrations and RSB analgesia in patients with midline laparotomy. 
The primary products of LP are lipid hydroperoxides (LOOH) $(1,2)$. Among the many different aldehydes which can be formed as secondary products during LP, MDA and 4$\mathrm{HNE}$ are receiving most attention because they are being considered as possible risk factor of cancer, cardiovascular diseases, chronic inflammation, diabetes, liver disease, Parkinson's disease, and respiratory distress syndrome $(1,2)$. In addition, LP products have been suggested as a possible risk factor for Alzheimer's disease $(1,2)$. In everyday life, attention must be paid to household oils used regularly in caterings and cooking, because in those processes very high amounts of LP products are generated and they can be easily absorbed through the diet $(1,2)$. The production and plasma level of MDA and 4-HNE can be measured and used as a biomarker to estimate the degree of oxidative stress and lipid peroxidation in human disease $(1,2)$. Both are toxic substances reacting with deoxyadenosine in DNA and forming DNA adducts, which are mutagenic $(1,2)$. The aldehydes which can be formed as secondary products during LP have been reviewed by Ayala et al. (2), where MDA is shown to be the most mutagenic product of LP, whereas 4-HNE is the most toxic product (2).

One possible bias of the study is that the patients in the control group and in the single-dose group had significantly higher mean body weight and body mass index than patients in the repeated-dose and continuous-infusion group. Unfortunately, we have no explanation for this trend, and due to the small sample size $(n=44)$, its significance should be very carefully interpreted. Despite that, following surgery, the RSB study groups did not differ in terms of NRS pain at rest and pain under $2 \mathrm{~kg}$ pressure to the wound area. However, the statistically significant inverse correlation between the individual plasma 4-HNE values and NRS pain at rest scores might suggest some role for 4 -HNE. In addition, $\mathrm{SFS}_{24}$ differed significantly between study groups, the repeated-dose (score 9.5) and continuous-infusion group (score 10) patients having highest $\mathrm{SFS}_{24}$ scores $24 \mathrm{~h}$ following surgery.

Although, the $\mathrm{NRS}_{\mathrm{r}}$ correlated significantly to 4-HNE plasma concentrations $(p=0.026)$, there was no statistically significant difference shown between plasma 4-HNE concentrations and the $\mathrm{NRS}_{\mathrm{r}} / \mathrm{NRS}_{\mathrm{p}}$ and $\mathrm{SFS}_{24}$ scores in the control and three RSB study groups. Interestingly, in the present study there was a significant decrease in 4-HNE concentrations following surgery in benign and cancer patients. In addition, the time effect in the linear mixed model in plasma 4-HNE was statistically significant. Saimanen et al. $(12,13)$ reported earlier that RSB-analgesia significantly increases the CAT concentrations and decreases the NT concentrations (20) immediately after operation. Surprisingly CAT concentrations dropped back $24 \mathrm{~h}$ postoperatively. Kärkkäinen et al. (15) reported earlier that RSB-analgesia does significantly increase the SOD-1 concentrations immediately after operation, but SOD-1 concentration drops back 24 h postoperatively. Martins et al. (21) have previously demonstrated that accumulation of
4-HNE plays a major role in the establishment and progression of pain showing that the 4-HNE is an endogenous aldehyde generated during oxidative stress, mainly through the LP process in the mitochondria. Therefore, increased 4-HNE adducts in the injured tissue positively correlates with pain (21). Sauer et al. (22) showed that the Delphinidin (DEL), a plant-derived antioxidant with potential to treat inflammatory pain, prevented 4-HNE-induced mechanical hyperalgesia, cold allodynia, and an increase in the intracellular calcium concentration into transient receptor potential ankyrin 1 expressing cells. The aim of their study was to develop a welltolerated cyclodextrin (CD)-DEL complex with enhanced bioavailability and to investigate the mechanisms behind its antinociceptive effects in a preclinical model of inflammatory pain, and formation of the LP product 4-HNE.

\section{Conclusion}

The enhanced ROS production alters the mitochondrial genome and proteome function through the accumulation of LP products, such as 4-HNE and MDA. Some mitochondrial protein products (e.g., Sirtuin3, SIRT3) can reduce ROS levels by modulating key antioxidant enzymes, such as manganese superoxide dismutase (MnSOD) (23). The present study showed that plasma 4-HNE concentrations decreased significantly after operation in all patients and 4-HNE concentrations were inversely correlated to the overall pain at rest (the $\mathrm{NRS}_{\mathrm{r}}$ pain scores). The present study confirms the applicability of the plasma biomarker 4-HNE to cast further light on the postoperative pain in midline laparotomy patients.

\section{Conflicts of Interest}

The Authors report no conflicts of interest or have any financial ties to disclose.

\section{Authors' Contributions}

The Authors M.E., I.S., R.K, A.H., T.S. and M.E. did the collection and analysis of data. M.E., I.S., R.K, A.H., T.S. and M.E. drafted and revised the manuscript, read and approved the final article.

\section{Acknowledgements}

The study was funded by the Heikki, Aino and Aarne Korhonen Foundation, Kuopio, Finland, and the Päivikki and Sakari Sohlberg Foundation, Helsinki, Finland.

\section{References}

1 Bartsch $\mathrm{H}$ and Nair J: Chronic inflammation and oxidative stress in the genesis and perpetuation of cancer: role of lipid peroxidation, DNA damage, and repair. Langenbecks Arch Surg 391(5): 499-510, 2006. PMID: 16909291. DOI: 10.1007/s00423006-0073-1 
2 Ayala A, Muñoz MF and Argüelles S: Lipid peroxidation: production, metabolism, and signaling mechanisms of malondialdehyde and 4-hydroxy-2-nonenal. Oxid Med Cell Longev 2014: 360438, 2014. PMID: 24999379. DOI: 10.1155/2014/360438

3 Purdy M, Kokki M, Anttila M, Aspinen S, Juvonen P, Selander T, Kokki H, Pulkki K and Eskelinen M: Does post-surgery placement of rectus sheath block analgesia alter the oxidative stress biomarker 8-OHdG concentrations: a randomised trial of patients with cancer and benign disease. Cancer Genomics Proteomics 13(3): 239-244, 2016. PMID: 27107066.

4 Purdy M, Kärkkäinen J, Kokki M, Anttila M, Aspinen S, Juvonen P, Kokki H, Pulkki K, Rantanen T and Eskelinen M: Does rectus sheath block analgesia alter levels of the oxidative stress biomarker glutathione peroxidase: a randomised trial of patients with cancer and benign disease. Anticancer Res 37(2): 897-902, 2017. PMID: 28179349. DOI: 10.21873/anticanres.11396

5 Bakirezer SD, Yaltirik CK, Kaya AH, Yilmaz SG, Ozdogan S, Billur D and Isbir T: The evaluation of glutathione reductase and malondialdehyde levels in patients with lumbar disc degeneration disease. In Vivo 33(3): 811-814, 2019. PMID: 31028201. DOI: 10.21873 /invivo.11543

6 Dogaru G, Bulboaca A, Boarescu PM, Ciumarnean L, Rus V, Sitar-Taut AV, Munteanu C, Bodisz G and Stanescu I: The effect of mofettes on oxidative stress/antioxidant balance in experimental myocardial ischemia. In Vivo 33(6): 1911-1920, 2019. PMID: 31662519. DOI: 10.21873/invivo.11685

7 Tsounapi P, Honda M, Hikita K, Sofikitis N and Takenaka A: Oxidative stress alterations in the bladder of a short-period type 2 diabetes rat model: antioxidant treatment can be beneficial for the bladder. In Vivo 33(6): 1819-1826, 2019. PMID: 31662508. DOI: 10.21873 /invivo.11674

8 Sardaro N, Della Vella F, Incalza MA, DI Stasio D, Lucchese A, Contaldo M, Laudadio C and Petruzzi M: Oxidative stress and oral mucosal diseases: an overview. In Vivo 33(2): 289-296, 2019. PMID: 30804105. DOI: 10.21873/invivo.11474

9 VON Woedtke T, Schmidt A, Bekeschus S, Wende K and Weltmann KD: Plasma medicine: a field of applied redox biology. In Vivo 33(4): 1011-1026, 2019. PMID: 31280189. DOI: 10.21873 /invivo. 11570

10 Carini F, Mazzola M, Rappa F, Jurjus A, Geagea AG, Al Kattar S, Bou-Assi T, Jurjus R, Damiani P, Leone A and Tomasello G: Colorectal carcinogenesis: role of oxidative stress and antioxidants. Anticancer Res 37(9): 4759-4766, 2017. PMID: 28870894. DOI: 10.21873 /anticanres.11882

11 Mokbel K and Mokbel K: Chemoprevention of breast cancer with vitamins and micronutrients: a concise review. In Vivo 33(4): 983997, 2019. PMID: 31280187. DOI: 10.21873/invivo.11568

12 Saimanen I, Kuosmanen V, Kärkkäinen J, Selander T, Aspinen $\mathrm{S}$, Holopainen $\mathrm{A}$, Rantanen $\mathrm{T}$ and Eskelinen $\mathrm{M}$ : Cholecystectomy patients with high plasma level of catalase have significantly lower analgesia requirement: a prospective study of two different cholecystectomy techniques with special reference to patients with cancer. Anticancer Res 38(9): 54175422, 2018. PMID: 30194197. DOI: 10.21873/anticanres.12872

13 Saimanen I, Kärkkäinen J, Selander T, Purdy M, Kokki M, Kokki $\mathrm{H}$ and Eskelinen M: Plasma catalase in relation to pain following midline laparotomy: a prospective study of patients with benign diseases and patients with cancer. Anticancer Res 38(11): 64796484, 2018. PMID: 30396975. DOI: 10.21873/anticanres.13011

14 Kärkkäinen J, Aspinen S, Harju J, Juvonen P, Pulkki K and Eskelinen M: Plasma glutathione peroxidase (GPX1) levels and oxidative stress in gallstone patients operated with two different cholecystectomy techniques: a randomized study with special reference to cancer patients. Anticancer Res 37(12): 6921-6927, 2017. PMID: 29187474. DOI: 10.21873/anticanres.12156

15 Kärkkäinen J, Selander T, Purdy M, Juvonen P and Eskelinen M: Patients with increased levels of the oxidative stress biomarker SOD1 appear to have diminished postoperative pain after midline laparotomy: a randomised trial with special reference to postoperative pain score (NRS). Anticancer Res 38(2): 1003-1008, 2018. PMID: 29374733. DOI: 10.21873/anticanres.12315

16 Kärkkäinen J, Saimanen I, Selander T, Aspinen S, Harju J, Juvonen P and Eskelinen M: Gallstone patients with enhanced oxidative stress biomarker superoxide dismutase (SOD1) plasma levels have significantly lower number of postoperative analgesic oxycodone doses: a prospective study with special reference to cancer patients. Anticancer Res 38(6): 3573-3578, 2018. PMID: 29848712. DOI: 10.21873/anticanres.12630

17 Luongo M, Brigida AL, Mascolo L and Gaudino G: Possible therapeutic effects of ozone mixture on hypoxia in tumor development. Anticancer Res 37(2): 425-435, 2017. PMID: 28179287. DOI: 10.21873/anticanres.11334

18 Wakana H, Kono H, Fukushima H, Nakata Y, Akazawa Y, Maruyama S, Hagio K, Fujii $\mathrm{H}$ and Ichikawa D: Effects of medium-chain triglycerides administration in chemically-induced carcinogenesis in mice. Anticancer Res 39(12): 6653-6660, 2019. PMID: 31810930. DOI: 10.21873/anticanres.13880

19 DE Angelis PM, Dorg L, Pham S and Andersen SN: DNA repair protein expression and oxidative/nitrosative stress in ulcerative colitis and sporadic colorectal cancer. Anticancer Res 41(7): 32613270, 2021. PMID: 34230120. DOI: 10.21873/anticanres.15112

20 Kuosmanen V, Saimanen I, Rahkola D, Kärkkäinen J, Selander T, Purdy M, Kokki H, Kokki M and Eskelinen M: Rectus sheath block (RSB) analgesia could enhance significantly the patient satisfaction following midline laparotomy in benign disease and in cancer: a prospective study with special reference to nitrosative stress marker nitrotyrosine (NT) plasma concentrations. Anticancer Res 39(3): 1383-1389, 2019. PMID: 30842172. DOI: 10.21873 /anticanres.13252

21 Martins BB, Hösch NG, Alcantara QA, Budas GR, Chen CH, Mochly-Rosen D, Ferreira JCB and Zambelli VO: Activation of PKCE-ALDH2 axis prevents 4-HNE-induced pain in mice. Biomolecules 11(12): 1798, 2021. PMID: 34944441. DOI: 10.3390/biom11121798

22 Sauer RS, Krummenacher I, Bankoglu EE, Yang S, Oehler B, Schöppler F, Mohammadi M, Güntzel P, Ben-Kraiem A, Holzgrabe U, Stopper H, Broscheit JA, Braunschweig H, Roewer N, Brack A and Rittner HL: Stabilization of delphinidin in complex with sulfobutylether- $\beta$-cyclodextrin allows for antinociception in inflammatory pain. Antioxid Redox Signal 34(16): 1260-1279, 2021. PMID: 32977733. DOI: 10.1089/ars.2019.7957

23 Ilari S, Giancotti LA, Lauro F, Gliozzi M, Malafoglia V, Palma E, Tafani M, Russo MA, Tomino C, Fini M, Salvemini D, Mollace $\mathrm{V}$ and Muscoli C: Natural antioxidant control of neuropathic pain-exploring the role of mitochondrial SIRT3 pathway. Antioxidants (Basel) 9(11): 1103, 2020. PMID: 33182469. DOI: $10.3390 /$ antiox9111103

Received January 21, 2022

Revised February 8, 2022 Accepted February 15, 2022 